\title{
Summary.
}

I. The spark spectra and the arc spectra of gallium, indium and zinc have been studied, and the delicacy of the spectroscopic detection of gallium and indium alone and in the presence of each other ascertained. Gallium chloride prepared by double distillation is found to contain less than $0.005 \%$ of either indium or zinc.

2. The purification of gallium by fractional electrolysis, and the preparation of pure gallium chloride by distillation are described.

3. Methods for the determination of gallium alone and of gallium, indium, zinc and aluminum in the presence of one another have been developed.

4. Two new salts of gallium, gallium selenate, and caesium gallium selenate alum, have been prepared. The solubilities of ammonium gallium sulfate alum and of caesium gallium sulfate alum in water, in $50 \%$ alcohol and in $70 \%$ alcohol, have been measured.

ITHACA, N. Y.

[CONTRIBUTION FROM THE CheMICAL LABORATORY OF MCGILL UNIVERSITY.]

\section{UNSATURATION AND MOLECULAR COMPOUND FORMATION.}

By O. MaAss and J. Russeli.

Received August 2, 1918.

It has been shown that organic substances containing oxygen, such as the alcohols and ethers, form additive compounds with the halogens and halogen hydrides. The hypothesis put forward to account for the existence of these compounds is based on the supposed tetravalency of oxygen and the consequent formation at low temperatures of compounds, commonly called oxonium compounds.

In the course of an investigation ${ }^{1}$ of these oxonium compounds several systems of non-oxygen-containing substances and the halogen hydrides were examined with the object of showing that, in the absence of oxygen, compounds are not formed. The system chloroform-hydrobromic acid illustrated this. But contrary to expectations toluene was found to form an addition compound with hydrobromic acid similar to an oxonium compound, and further experiments showed that toluene and chlorine combine to form such compounds. The present investigation has been carried out with the object of ascertaining the nature of these toluene compounds by determining whether aromatic and unsaturated hydrocarbons in general combine in a similar manner.

Modern theories of molecular compound formation are reverting more and more to a modified form of Berzelius' views. Molecules are supposed to combine in virtue of residual forces which, too small to hold the molecules together at high temperatures, come into play at low temperatures

1 This Journal, 34, I273 (I912). 
where the translatory movement of the molecules is so much smaller. The residual force which causes the formation of oxonium compounds may be due to the 4 valences of oxygen, two of which may be supposed to be pronounced and two relatively weak. The latter then give to the molecule, of which the oxygen atom forms a part, the power to form an additive compound at a low temperature.

Toluene is composed of hydrogen and carbon, two elements whose valences are especially well defined and invariable. Its power to form an additive compound must be accounted for in some other way. The unsaturated linkages suggest themselves as the possible source of residual valency. This idea is not a new one; Norris, ${ }^{1}$ for instance, suggested that unsaturation was the cause of the formation of some addition compounds which he investigated.

It might be well to refer to a paper ${ }^{2}$ on aromatic oxonium compounds wherein it was shown that these compounds are very complex, for instance $\mathrm{C}_{6} \mathrm{H}_{5} \mathrm{COC}_{6} \mathrm{H}_{5} .6 \mathrm{HCl}$. To account for the formation of such compounds with oxygen as the critical factor would require the supposition of hexavalent, and even octavalent, oxygen. In view of the compounds which toluene forms the complexity of the aromatic halogen oxonium compounds may be in part due to the residual valency of the benzene nucleus.

The above outline may suffice to show the value of a research confined to investigating the effect of the structure of a hydrocarbon on additive compound formation. With this object in view it was thought desirable to examine the following systems: benzene, toluene, ethyl-benzene, mesitylene, the xylenes, acetylene, allylene and ethane; hydrobromic acid to be the second component in every case.

The principle of the experimental method was the ordinary one of measuring the freezing points of various concentrations of hydrocarbonhydrobromic acid. Many experimental difficulties had to be overcome, and, as these varied from system to system, no general description can be given, but the special methods used in examining each system may best be outlined separately, together with the results obtained.

The temperatures throughout were measured by means of a platinum resistance thermometer consisting of a coil of fine platinum wire fused into a quartz rod. The variable resistance in the Wheatstone bridge was an accurately calibrated Kohlrausch bridge, making it possible to read the temperature to $0.1^{\circ}$. The freezing points quoted, however, may be taken as accurate to only $I^{\circ}$, this being the error involved in observing the disappearance of the solid phase. This was deemed sufficiently accurate, as the relative variation of freezing point with concentration is quite large.

1. This Journal, 38, 702 (1916).

2 Ibid., 33, 7I (I9II). 
The hydrobromic acid was prepared and purified in the ordinary way, liquefied by means of solid carbon dioxide-ether mixture, and redistilled through phosphorus pentoxide tubes into the apparatus. To determine the desired percentage composition of the hydrocarbon-hydrobromic acid mixture the weight method, described in detail in a previous paper, ${ }^{1}$ was employed wherever possible.

In the case of benzene, the first system to be examined, the weight method was employed for concentrations of $50 \%$ to $100 \%$ acid. At lower concentrations the freezing points were so high that hydrobromic acid boiled off from the mixture so that resource was had to the following manipulation. A small bulb was blown at the end of a small glass tube. This was weighed, the bulb partly filled with benzene and reweighed. The bulb was then immersed in the solid carbon dioxide-ether mixture and hydrobromic acid distilled in by means of fine capillary tubes. When sufficient hydrobromic acid was thought to have been added, the delivery tubes were withdrawn, a cork inserted and the tube reweighed. If the percentage was about where a point was desired on the curve the next stage was begun. If not, more hydrobromic acid was distilled into it, using, of course, fresh capillaries. When a satisfactory percentage had been obtained a stiff wire was attached just above the bulb and by means of a small blow-pipe flame the bulb was sealed off. The stiff wire was for the purpose of holding the bulb, the lower portion of which was immersed in solid carbon dioxide-ether mixture while being sealed off. The mixture in the bulb was then allowed to warm up, the contents to mix thoroughly and the freezing-point determined by a method to be outlined presently. After the freezing-point was determined the wire was removed from the bulb and the latter was weighed accurately. The long entrance tube formerly attached to it was also weighed accurately and thus the amount of hydrobromic acid exactly determined. The freezing points of the contents of these bulbs were redetermined some weeks later and found not to have changed. To check the percentages the mixtures were analyzed as follows: The bulb, previously weighed, was immersed in carbon dioxideether mixture and the tip cut off; it was then dropped into a large Erlenmeyer flask filled with distilled water, and covered. The hydrobromic acid was absorbed by the water and determined by standard alkali. The bulb itself with the tip which was broken off was carefully dried and weighed accurately. This analysis gave results concordant with the percentages as determined by weight, as the following figures taken from the first few samples will indicate.

Percentages.

\begin{tabular}{|c|c|c|c|c|}
\hline By weight. & 27.01 & 38.21 & $33.6 \mathrm{I}$ & $42 \cdot 38$ \\
\hline 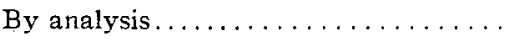 & 27.05 & 38.13 & 33.68 & 42.02 \\
\hline
\end{tabular}


The results by analysis were probably the more accurate as there was less opportunity for the entrance of such disturbing factors as the evaporation of the benzene; they were therefore taken as the true percentages.

In order to measure the freezing points of the mixtures in the bulbs, use was made of a cooled ether bath contained in an unsilvered Dewar flask. The temperature of the ether bath could be lowered by the addition of a few lumps of solid carbon dioxide and raised by the addition of a little more ether. A continuous stream of air bubbling through the bath kept it stirred and maintained a uniform temperature, as registered by the thermometer immersed in it. This arrangement makes it possible to keep the bath at any desired temperature between the limits $0^{\circ}$ and $-75^{\circ} .{ }^{1}$ The bulb held by the wire was placed in the bath and its contents thoroughly stirred by shaking and turning it. By changing the

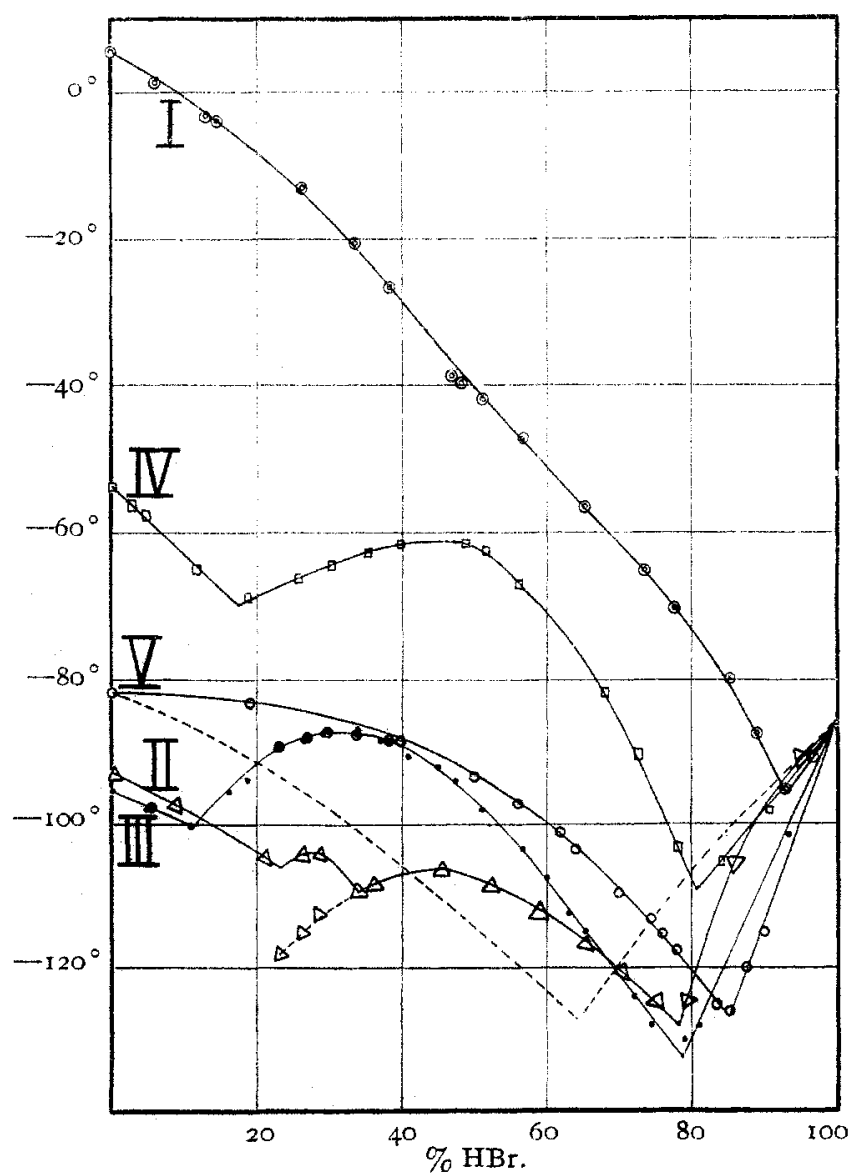

Fig. I.

1 This Journat, 35, 536 (1913). 
temperature of the bath the point was obtained, at which the last traces of solid in the bulb did not grow on being left for some time; this was a minimum. Now the solid was completely melted by warming up the bath a few degrees, and the bath was then cooled by stages till a point was reached at which the solid just disappeared. This was taken as the freezing point of the mixture. The difference between the minimum point obtained and the first point after warming the bath was only one or two degrees, so that the points were determined with sufficient accuracy.

The values obtained for the freezing points of the benzene-hydrobromic acid system are given in Table I and represented in Fig. I, Curve I. It will be seen that no compound is formed between these two substances, as there is no suggestion of a maximum at $50.1 \%$ acid, corresponding to a one-to-one compound or $36.6 \%$ corresponding to a compound two benzene to one acid.

TABLE I.

Freezing Points of the Benzene-Hydrobromic Acid System.

$\%$ acid by weight.

0

$5 \cdot 9$

I 2.9

I 4.5

27.05

33.6

38.2

42.0

46.6

48.02

51.0

57 . I

65.2

73.5

77.8

85.7

88.97

92.96

96.13

100.0
Freezing temperature. Degrees C.

$5 \cdot 4$

I. 3

$-3.5$

$-4.0$

$-12.5$

$-20.5$

$-26.5$

$-31.0$

$-39.0$

$-40.0$

$-42.0$

$-47.0$

$-56.5$

$-65.0$

$-70.5$

$-79.5$

$-87.5$

$-95.0$

$-90.0$

$-86.0$

Since a compound forms between toluol and hydrobromic acid and yet no combination occurs between benzene and the acid, it was thought that ethyl benzene would logically be the next hydrocarbon to investigate. Much difficulty was encountered in its preparation due to lack of chemicals but finally a very pure sample was obtained from the laboratories of the University of Illinois; its boiling point was $136.5^{\circ}$ and its melting point $-92.4^{\circ}$. With this substance as with the benzene, the slight cloudiness visible on cooling, due to minute traces of water, could only be removed by prolonged standing over sodium. The treatment of the ethyl benzene 
was precisely similar to that of benzene with the exception of the use of bulbs. This was not necessary as the hydrobromic acid is soluble in the hydrocarbon at ordinary pressures. It is interesting to note that the acid is also fairly soluble in toluene and mesitylene, both of which form compounds, while in benzene, which does not form a compound, it is relatively insoluble. By additions of the hydrocarbon to the acid, points on the curve were obtained from $100 \%$ acid to $26.3 \%$ acid. The last two points were very difficult to determine as the metastable phase would persist in coming out. Its melting point, of course, was at a much lower temperature. By repeated trials the stable higher melting phase was also obtained. Thus for a short period there is a metastable prolongation of one section of the curve. The remainder of the curve was completed by additions of the acid to the hydrocarbon. Liquid air was necessary for cooling purposes since temperatures as low as - $30^{\circ}$ were required.

The values obtained are given in Table II and are represented by Fig. I, Curve II. It will be noticed that here there are two compounds formed, one containing $43.3 \%$ acid corresponding to one ethyl benzene-one hydrobromic acid, melting at $-105.5^{\circ}$, the other corresponding to two ethyl benzene-one hydrobromic acid melting at - $103.8^{\circ}$ and containing $27.6 \%$ acid.

TABLE II.

Freezing Points of the Ethyl Benzene-Hydrobromic Acid System.

$\%$ acid by weight.

0

8.94

19.4

23.3

26.3

28.6

33.8

36.2

40.0

$45 \cdot 7$

52.4

58.9

$65 \cdot 4$

70.1

75.2

$79 \cdot 7$

$85 \cdot 2$

90.7

95.1

IOO.O
Freezing temperature.

Degrees C.

$--92.4$

$-97.7$

$-\operatorname{IO} 3.6$

... (- 118.5$)$

-104.0 (-115.2)

$-104.0(-112.6)$

-I I0. 2

$-109.0$

$-106.0$

$-106.3$

$-108.8$

$-112,2$

$-116.7$

$-120.5$

$-125.2$

- 125.0

—IO5.8

$-96.5$

$-90.8$

$-86.0$

The temperatures in brackets represent the temperatures on the metastable portion of the curve corresponding to the compositions indicated. 
The fact that ethyl benzene forms two compounds, one of which is a two to one compound, led to a closer investigation of the nature of the toluene-hydrobromic acid curve obtained by Maass and McIntosh. The maximum here corresponds to a compound-two toluene-one hydrobromic acid. However, it was decided to make check determinations on the part of the curve near the maximum to see if that was the true maximum. The experimental manipulation was similar to that in the previous curves, carbon dioxide-ether mixture under vacuum being used as the freezing agent. The values are represented in Table III and in Fig. I, Curve III are indicated in their relation to the values obtained by Maass and McIntosh. The values at the maximum are seen to correspond very closely, indicating a compound of the constitution two toluene -one hydrobromic acid, melting at $-86.5^{\circ}$ and containing $30.5 \%$ acid.

TABLE III.

Freezing Points of the Toluene-Hydrobromic Acid System.

$\%$ acid by weight.

6.7

23.6

26.8

29.5

34.8

39.9

$43 \cdot 7$

90.2

92.7

$95 \cdot 1$
Freezing temperature.
Degrees C.

$-96.6$

$-89.0$

$-88.3$

$-87.6$

$-88.0$

$-89.0$

$-90.6$

$-\mathrm{IOI} .5$

$-95.8$

$-91.6$

Continuing the series it was thought that the dimethyl substituted benzene ring might be very interesting. But at the time the pure xylenes had not as yet been prepared. The symmetrical trimethyl substituted benzene derivative, mesitylene, was however obtained. The sample used was Kahlbaum's, purified by repeated distillation over sodium and allowed to stand for some days over sodium wire; its boiling point was I $64.5^{\circ}$ and its melting point $-53.5^{\circ}$. The procedure was identical with that in the case of toluene, carbon dioxide under vacuum being again resorted to for freezing purposes.

The values obtained are shown in Table IV and represented in Fig. I, Curve IV. It will be seen that there is a well-defined maximum corresponding to a compound, one mesitylene-one hydrobromic acid of composition $40.3 \%$ acid.

It might be interesting to point out that saturated solutions of hydrobromic acid in the substituted benzene compounds when allowed to stand for some time at room temperature give evidence of chemical interaction, contrary to the usual conception of the behavior of aromatic hydrocarbons towards halogen acids. Benzene here, too, exhibits its usual chemical 
inertia, a solution of hydrobromic acid in benzene remaining unchanged after months.

TABLE IV.

Freezing Points of the Mesitylene Hydrobromic Acid System.

$\%$ acid by weight.

0

$2 \cdot 7$

4.5

II 6

I 8.5

25.6

$30 \cdot 3$

35.0

40.3

4 I. 5

48.8

50.2

51.7

55.9

57.9

68.2

72.7

77.95

84.5

9 I. 8

100.0
Freezing temperature.

Degrees C.

$-53.5$

$-5^{6.5}$

$-57.5$

$-65.5$

$-68.5$

$-66.5$

$-64.5$

$-63.0$

$-61.5$

$-6 \mathrm{r} .7$

$-61.7$

$-62.2$

$-62.5$

$-67.5$

$-68.4$

$-82.0$

$-90.0$

$-103.5$

$-105.0$

$-98.5$

$-86.0$

The experimental treatment of the liquid hydrocarbons, with the exception of benzene, it has been seen, presented no outstanding difficulty. The mutual insolubility of the two components in the case of the benzene was overcome by the somewhat tedious yet very accurate bulb method. The case of acetylene, however, presented much more difficulty. The two components are gaseous at ordinary temperature, introducing the awkwardness attendant upon the handling of such substances. Furthermore, the melting point of acetylene is so low that to preserve it in the liquid or solid form the carbon dioxide-ether mixture had to be kept continuously under vacuum. The most cogent argument against using the ordinary method was furnished by the fact that at room temperature the two components may react with one another and thus while distilling one gas into the other their vapors would meet above the surface of the liquid and this phase would be contaminated, producing an irregularity in the results, this irregularity not being constant, but increasing with the number of new additions of gas made to the system.

The acetylene was generated by dropping water on calcium carbide covered with alcohol, passed through two wash-bottles containing chromic acid and potassium hydroxide, respectively, through a tube of phosphorus pentoxide and condensed in a large test-tube by means of carbon dioxide- 
ether mixture under vacuum. The chromic acid removed the phosphine which is as a rule present, the potassium hydroxide removed the carbon dioxide and the phosphorus pentoxide removed the water. The condensation by solid carbon dioxide under vacuum eliminated the nitrogen and carbon monoxide which are sometimes present in samples made by this method. The substance thus collected was redistilled through wash bottles containing fresh reagents and passed into a large gasometer. A sample taken from this was found to be absorbed without residue in saturated bromine water. The gasometer containing the pure product was connected to the apparatus represented in Fig. 2 at A. The hydrobromic acid was prepared in the usual way and distilled into the tube $B$, the delivery tube being sealed off at the end. $\mathrm{L}$ and $\mathrm{K}$ were large flasks

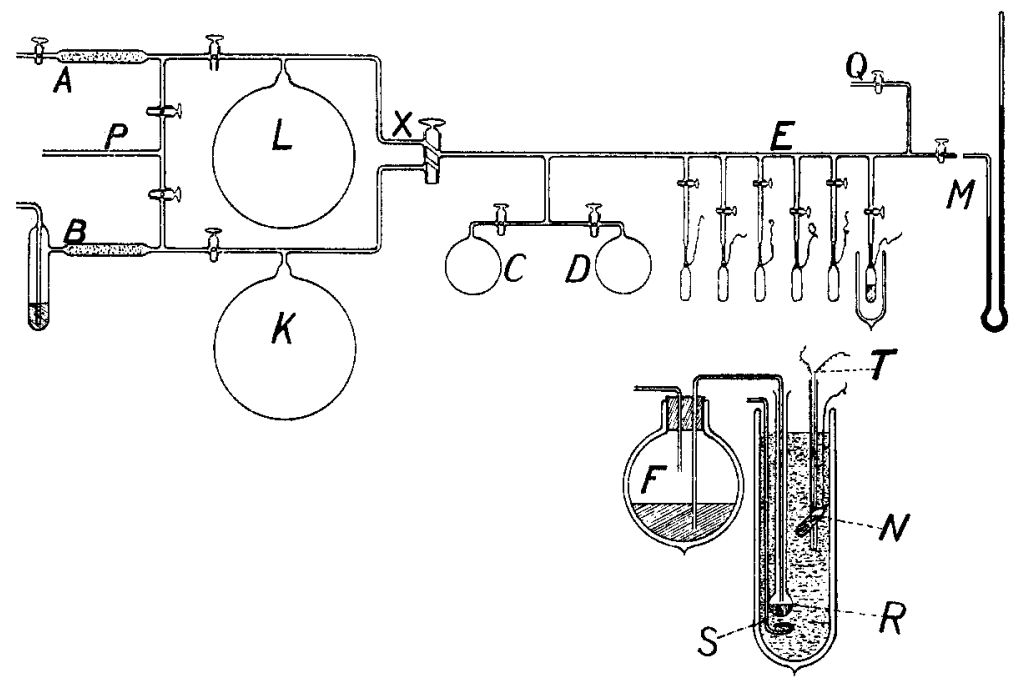

Fig. 2.

of approximately one-liter capacity, $\mathrm{C}$ and $\mathrm{D}$ smaller flasks whose volumes were about $\mathrm{I}_{50} \mathrm{cc}$. These volumes had been accurately calibrated as well as the volume of the tubing from the two-way tap at $\mathrm{X}$ to the manometer $\mathrm{M}$. At $\mathrm{E}$ a series of bulbs were arranged, $\mathrm{I} 2$ in number, only 6 being shown in the diagram. Immediately above each bulb there was a small constriction in the tubing and a little higher a tap by which it could be shut off from the system. All joints throughout the apparatus were of glass. The procedure was as follows: The taps on the tubing leading from the reservoir to $L$, were closed and the tap leading to the vacuum pump at $P$ opened, this section thus being exhausted. The tap connecting the system to the reservoir was opened once or twice to allow a rush of acetylene through the tubing, to wash out thoroughly any air in that section of tubing. Similarly the tap connecting $B$ to the vacuum pump $P$ was 
opened suddenly once or twice, thus drawing the air out of that section. Meanwhile the whole apparatus from the taps at $\mathrm{L}$ and $\mathrm{K}$ to the manometer $M$ had been completely evacuated by an automatic mercury pump connected at $Q$. Then the taps on the tubes leading to the bulbs at $\mathrm{E}$ and the bulbs at $\mathrm{C}$ and $\mathrm{D}$ were turned off. Acetylene was admitted to the large flask $\mathrm{L}$, till about atmospheric pressure was reached. It was then shut off from the reservoir and connected to the small bulb $C$. When equilibrium had been established the tap at $\mathrm{X}$ was closed and the pressure read as registered on the manometer at $M$. The tap was then opened to one of the bulbs surrounded by liquid air and the acetylene from the bulb $C$ thus condensed. A calculation had been made previously of the drop in pressure necessary to give the required amount of acetylene and as the mercury reached this height in the manometer the tap connecting the bulb to the system was closed. The volume formerly occupied by the gas was known and the change in pressure in the system: therefore the quantity condensed could be accurately calculated. ${ }^{1}$ The tap at $C$ was now closed, the tap at $Q$ being once more opened to exhaust thoroughly the tubing from $\mathrm{X}$ to $\mathrm{M}$. When this had been accomplished the pump was cut off by closing the tap, and the two-way tap at $\mathrm{X}$ opened to $\mathrm{K}$, which had been previously evacuated. Hydrobromic acid was then admitted to this flask by warming up the liquid in $\mathrm{B}$. The procedure now was the same as in the case of acetylene, except that the small bulb $D$ was used instead of $\mathrm{C}$, the acid being condensed on top of the hydrocarbon in the bulb. A calculation had been made beforehand of the change in pressure which would be requisite to give the desired percentage with the amount of acetylene already condensed, and the condensation of the acid was stopped at the right point by turning the tap at the head of the bulb. This was then sealed off at the constriction with a small blow-pipe flame and the freezing point of the mixture determined. For the handling of the bulb during the sealing off and after the sealing off, a stiff wire was attached in the following manner: a small strip of black adhesive tape was wound round the neck and round this the piece of stiff wire was bent, but not very tightly, on account of the danger of breaking the bulb off at the small construction above; it was then bound in place by fine, flexible wire.

Sometimes, to obtain the required amount of hydrocarbon or acid in the bulb, the small volume $\mathrm{C}$ or $\mathrm{D}$ would require to be filled from the large flask $\mathrm{L}$ or $\mathrm{K}$ one or two or more times. When the supply in $\mathrm{L}$ or $\mathrm{K}$ became very low, more could be obtained from the reservoir in the one case, or by warming up the liquid in the other.

At all times after the first condensation of the acetylene the bulb was

1 The density of the gases used had been determined by a method described elsewhere. 
kept immersed in liquid air, and thus when the acid was condensed on top of it at the temperature of the liquid air the solid hydrocarbon had no appreciable vapor pressure and thus there was no vapor which could react with the incoming acid vapor. The acid condensed as a solid and the two substances were kept in that condition until the bulb had been placed in the bath and had warmed up gradually to about $-90^{\circ}$, when for the first time the two components were allowed to mix thoroughly.

The freezing points of the mixtures in the bulbs were determined in. precisely the same way as those of benzene-hydrobromic acid. The low temperatures required in this case necessitated the use of liquid air to cool the bath, and to do this conveniently it was arranged as in the lower diagram in Fig. $2 ; \mathrm{N}$ and $\mathrm{T}$ represent the bulb and thermometer, respectively. $\mathrm{R}$ is a small bulb into which was led a tube from the Dewar flask containing the liquid air. Thus when desired to cool the bath, a little liquid air was introduced into this bulb. S is a tube from which issued a constant stream of dry air to keep the liquid of the bath well stirred and thus at a uniform temperature. The liquid used was in this case petroleum ether on account of its low melting point. With this arrangement the temperature of the bath was under complete control and by introducing from time to time just the right amount of liquid air into the bulb $\mathrm{C}$, the bath could be kept at constant temperature for almost any length of time desired. The values obtained from the various percentages are given in Table $V$, and are graphically represented in Fig. I, Curve V. It can be seen here that there is but one eutectic; that is to say, no compound formation occurs between the two components.

\section{TABLE V.}

Freezing Points of the Acetylene-Hydrobromic Acid System.

\% acid by weig
O
19.4
30.0
39.8
49.9
56.0
61.9
64.0
69.9
74.5
76.0
78.0
83.4
85.3
87.5
89.9
100.0

Molecular \% acid
O
7.2
I 2.9
I 5.4
24.2
28.4
34.3
36.1
42.8
48.4
50.0
53.2
61.7
65.6
69.4
74.3
I00.0

Freezing temp.

Degrees C.

$-81.8$

$-82.5$

$-85.7$

$-88.2$

$-93.2$

$-97.3$

-IOI.3

$-103.7$

$-109.6$

-II3.I

$-\mathrm{II} 5.2$

一I 7.6

-I 25.2

-1 26.0

- $\mathrm{I} 20.2$

-I 15.0

$-86.0$ 
The marked bend on the acetylene side of the curve is caused by the method of plotting, due to the small molecular weight of acetylene relative to that of hydrobromic acid. This is seen to disappear if the curve is plotted molecularly as indicated by the dotted line in Fig. I, Curve V.

Some interesting facts were noted as to the behavior of the bulbs when allowed to warm up to room temperature. Those whose contents were represented on the right side of the eutectic after a short time exploded violently. The remaining bulbs when broken some weeks later still contained large amounts of hydrobromic acid even though there was in the mixture a large excess of acetylene, that is to say, no interatomic combination between the substances had occurred at relatively high temperatures. In a previous attempt to determine the freezing-point curve of the two components a platinum stirrer was used, and it was noticed that a dark brown oil formed at the surface of the platinum, which oil persisted on the warming up of the mixture. It was thought that the platinum had catalyzed a reaction between the two components and therefore a bulb was made in the usual manner containing a piece of platinum. This bulb after a short time exploded with great violence, shattering to small pieces a large glass jar in which it was standing. This apparently indicates that the platinum had catalyzed the interatomic reaction between the components.

The precision of the method just described is well seen in the manipulation of the two gases, acetylene and hydrobromic acid, and the same apparatus is now being used for other systems where both components are gaseous.

To summarize the experimental work. Acetylene and benzene do not form molecular compounds with hydrobromic acid at low temperatures. The existence of a compound of the composition two toluene-one hydrobromic acid melting at $-86.5^{\circ}$ has been verified; compounds of the composition one ethyl benzene-one hydrobromic acid melting at $-105.5^{\circ}$, two ethyl benzene-one hydrobromic acid melting at -I03. $8^{\circ}$, one mesitylene-one hydrobromic acid melting at $-61.0^{\circ}$, have been shown to exist.

The authors hope to continue with the investigation of other systems. It is to be understood that the deductions based upon the described experiments may only be regarded as possible generalities, which require further experimental evidence.

With this proviso these deductions may briefly be summed up thus: An unsaturated bonding does not necessarily induce compound formation, as is exemplified by the behavior of benzene and acetylene. In these two substances the symmetrical arrangement of the linkages apparently establishes a stable equilibrium of the interatomic forces in which there is no externally directed force to exert an attraction upon hydro- 
bromic acid molecules. In the case of alkyl substitutions, this equilibrium of forces is disturbed so that molecular combination can take place.

Montreal, Canada.

[CONTRIBUTION FROM THE CHEMTCAL LABORATORY OF THE UNIVERSITY OF WASHINGTON.]

SALTS OF HELIANTHINE.

By Charlas R. Stark and William M. Dehn.

Received June 19, 1918.

From recent studies by one of us with methyl orange ${ }^{1}$ and with other colored solutes, ${ }^{2}$ the conclusion was drawn that color changes in solutions are largely or wholly independent of ionic $^{3}$ concentrations. An hypothesis, interpreting such color changes and embracing the following propositions, ${ }^{4}$ was indicated:

(I) The colored solute forms additive compounds with acids, with bases, or with "indifferent" solvents.

(2) Solutions of such additive compounds are more or less transformed by the solvent, yielding either simple chromoisomeric equilibria or polychromic equilibria.

(3) The intrinsic susceptibility of the additive compound to the transforming influence of the solvent is the important factor of color changes in solutions.

(4) Heat $^{5}$ and probably other physical conditions have secondary influences on color changes.

This paper will concern itself largely with the first proposition-that the colored solute forms additive compounds. It will be shown that helianthine forms salts with great ease and that all of these salts can be interpreted as additive compounds.

Prior to this only salts of sodium, ammonium, pyridine, ${ }^{6}$ and hydrochloric acid ${ }^{7}$ have been described. This paucity of described salts is remarkable, when it is observed that helianthine forms salts with the readiness of a strong acid, uniting with all classes of bases whether organic or inorganic.

Owing to its amphoteric nature, helianthine also forms salts with acids, but it was found that these salts cannot be prepared pure ${ }^{7}$ in aqueous solutions. However, if the isomerizing influence of water is avoided, that is, if anhydrous conditions are observed, violet-red salts, compounded

1 This Journal, 39, 1348, 1377 (1917).

2 Ibid., 36, 846 (1914); 39, 1338, 1381, 1392, 1399 (1917).

3 Ibid., 39, 1357, 1390, 1396 (1917).

4 Ibid., 39, 1376, 1380, 1403 (1917).

- Ibid., 36, 845 (1914); 39, 1374, I391 (1917).

- Hantzsch, Ber., 46, 1538 (1913).

7 Hantzsch, Ibid., 4I, I 192 (1908). 